\title{
AVALIAÇÃO DE DESEMPENHO TÉRMICO DE UMA RESIDÊNCIA ATRAVÉS DE SIMULAÇÕES COM O ENERGYPLUS
}

Thermal performance evaluation of a residence through EnergyPlus simulations http://dx.doi.org/10.21116/2019.5

\section{TURCZYN, Daniel Teixeira}

Universidade Estadual de Campinas

\author{
NEVES, Letícia de Oliveira \\ Universidade Estadual de Campinas
}

RESUMO: O desempenho térmico das edificações está diretamente associado aos fatores geográficos e climáticos do local de inserção e das escolhas de formas e materiais feitas pelos projetistas. Uma das formas de avaliar o conforto térmico dos usuários de uma edificação é através do modelo adaptativo para ambientes naturalmente ventilados presente na ASHRAE55, no qual é possível definir os limites máximos e mínimos da zona de conforto e averiguar as condições da temperatura operativa interna do ambiente. Um dos modos de conseguir as temperaturas operativas internas é através de simulações computacionais no programa EnergyPlus. A partir da averiguação do desempenho térmico de uma edificação é possível estudar comparativamente soluções alternativas de projeto e de escolha de materiais, buscando soluções que aumentem o período de conforto sem a necessidade da utilização de sistemas elétricos. O objetivo deste trabalho é avaliar o desempenho térmico dos ambientes de estar e social de uma residência unifamiliar localizada no município de Campinas, bem como das alternativas de projeto e de materiais construtivos propostos para a redução do período de desconforto. A avaliação desenvolvida apontou o total de 2.574 graus-hora de desconforto no ano para o período de ocupação (14-22h). Foram realizadas vinte e quatro simulações alternativas para esses ambientes, alterando as camadas e os materiais construtivos que conformam a cobertura, o piso, os vidros, as paredes internas e os elementos de proteção solar, bem como três simulações que mesclaram essas alterações. Os resultados apontam que é possível reduzir a carga de desconforto de diversas maneiras, desde soluções baratas e simples, como pintar a telha de branco e diminuir a espessura da laje de piso, até soluções com materiais mais específicos e custosos, como o uso de vidros insulados.

Palavras-chaves: Desempenho Térmico; Eficiência Energética; EnergyPlus

\begin{abstract}
The building thermal performance is directly associated with the geographical and climatic factors of the place and the choices made by designers. A method of evaluate the thermal comfort of a building occupancy is through the adaptive model for naturally conditioned spaces present in ASHRAE55. It enables the definition of the maximum and minimum limits of the comfort zone and to verify the conditions of the internal operating temperature of the environment. One way to achieve internal operating temperatures is through computer simulations in the EnergyPlus software. The investigation of the building thermal performance makes possible the study of alternatives of design and materials, seeking solutions that increase the period of comfort without the
\end{abstract}


use of electrical systems. The aim of this work is to evaluate the thermal performance of the living and social environments of a single-family dwelling located in the city of Campinas, as well as the alternatives of design and construction materials proposed to reduce the period of discomfort. The evaluation developed indicated a total of 2,574 discomfort degree hours in the year for the period of occupation (14-22h). Twenty-four alternative simulations were performed for these spaces, changing the layers and construction materials that make up the roof, floor, windows, internal walls and sun protection elements, as well as three simulations that blended these alternatives. The results show that it is possible to reduce the discomfort load in different ways, from cheap and simple solutions such as white tile painting and thinning of the floor slab, to solutions with more specific and expensive materials such as the use of glass insulated.

Key-words: Thermal Performance, Energy Efficiency; EnergyPlus

\section{INTRODUÇÃO}

O desempenho térmico de uma edificação depende de diferentes fatores, desde as características que são intrínsecas do local - como a localização geográfica, as características climáticas, a forma urbana etc. - até as características formais e materiais do projeto, que dependem da escolha de um projetista - como o posicionamento da edificação no terreno, os materiais que compõem as vedações opacas externas (piso, paredes e cobertura) e internas (paredes e portas), os vidros que integram as vedações transparentes (janelas), os sistemas de proteções solares etc. Mudar um ou alguns desse fatores pode acarretar na alteração do desempenho térmico dessa edificação, diminuindo ou aumentando o período de desconforto.

Avaliar o desempenho térmico de uma edificação consiste na verificação da resposta térmica desta quando em contato com o meio externo, verificando se o ambiente construído interno apresenta condições térmicas suficientes para o conforto das pessoas. Um dos modos de fazer essa avaliação é através do modelo adaptativo para ambientes naturalmente ventilados, proposto por DeDear e Brager (2002) e incorporado na ASHRAE55 de 2004. O modelo define uma equação que determina a temperatura de neutralidade do mês e, a partir dela, é possível definir os limites máximos e mínimos de conforto. Para Pereira e Assis (2010) o modelo adaptativo é um modo simplificado de fazer essa averiguação, apresentando uma temperatura de conforto térmico em função da temperatura média mensal registrada, e aponta que é um modelo exploratório 
eficiente para as etapas iniciais do projeto arquitetônico, nas quais o projetista possui poucos dados e precisa de informações objetivas para direcionar suas estratégias de projeto.

Com o auxílio de ferramentas de simulação termoenergética é possível verificar quais períodos do ano ou quais horas dos dias apresentam condições críticas ao conforto, ou seja, que estão fora do limite de conforto aceitável. Um dos dados possíveis de conseguir com as simulações computacionais é a temperatura operativa interna do ambiente, pois a partir dela é possível averiguar se o ambiente está dentro ou fora da zona de conforto delimitada através do modelo adaptativo supracitado. Se a temperatura operativa interna for superior ao limite máximo de conforto, soma-se os graus-hora de resfriamento, se for inferior ao limite mínimo de conforto, soma-se os graus-hora de aquecimento. A soma dos graus-hora de resfriamento e de aquecimento resulta na quantidade de graus-hora de desconforto do ambiente.

A partir dessa averiguação é possível estudar diferentes alternativas de projeto, buscando as soluções que resultem na menor quantidade de graus-hora de desconforto e, consequentemente, no menor consumo de energia com o uso de sistemas ativos de condicionamento de ar. As alternativas passíveis de serem estudadas estão relacionadas com os diferentes fatores que determinam o desempenho térmico da edificação, por exemplo, é possível mudar a orientação do prédio, a composição das camadas da cobertura, os materiais de vedação, os tipos de vidros etc., e quantificar quanto cada alteração reduz a carga de desconforto do ambiente.

\section{OBJETIVO}

O objetivo deste trabalho é avaliar o desempenho térmico dos ambientes de estar e social de uma residência unifamiliar localizada no município de Campinas (SP), bem como das alternativas de projeto e de materiais construtivos propostos para a redução do período de desconforto.

\section{MÉTODO}




\subsection{Objeto de Estudo}

O objeto de estudo do trabalho foi o ambiente de estar e social de uma residência unifamiliar térrea localizada no município de Campinas, no estado de São Paulo, com latitude de $-22,82^{\circ}$, longitude de $-47,06^{\circ}$ e altitude de $640,0 \mathrm{~m}^{2}$. A residência tem área construída de $330 \mathrm{~m}^{2}$ e a área analisada tem $105,0 \mathrm{~m}^{2} \mathrm{com}$ um pé direito de 3,0m (Figura 5). A maior face do ambiente analisado é voltada para o exterior e tem orientação para o oeste, com um percentual de abertura da fachada (PAF) de $82 \%$, já a face sul é voltada para o quintal da residência, com um PAF de 95\%, ou seja, o ambiente possui grandes aberturas para o exterior, sendo a maior delas para uma orientação solar desfavorável. As janelas voltadas para o oeste possuem um brise diferenciado que mescla elementos verticais (ângulo $\beta$ ) e elementos de sombreamentos paralelos ao vidro, chamados aqui de sombreamento frontal. Como o brise e o beiral da fachada oeste não estão presentes em toda a sua extensão a face foi dividida em três partes, para facilitar a simulação dos brises (Figura 6).

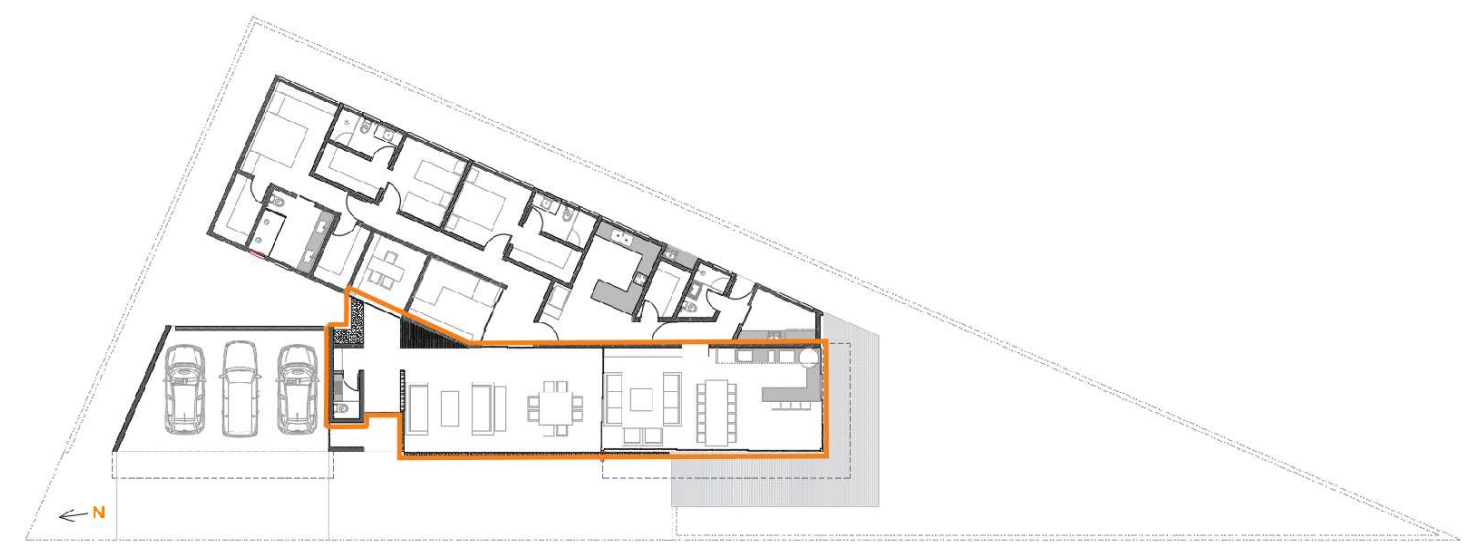

Figura 5- Planta sem escala da residência, com a demarcação em laranja dos ambientes estudados. Fonte: produzido pelos autores.

2 Os dados geográficos de Campinas foram retirados do arquivo EPW disponibilizado pelo Labeee da UFSC, disponível em http://www.labeee.ufsc.br/downloads/arquivos-climaticos/inmet2016, acessado dia 26/09/2018. 


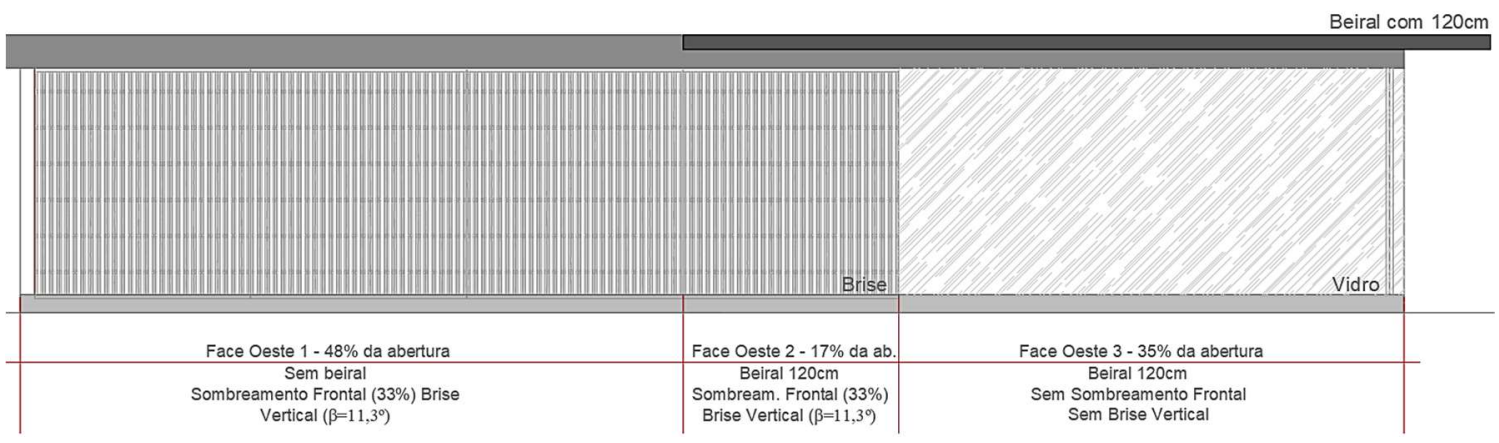

Figura 6- Desenho esquemático da parte da fachada oeste que tem as aberturas e brises, mostrando a divisão feita para facilitar a análise dos sistemas de proteção solar. Fonte: produzido pelos autores.

\subsection{Simulação Computacional}

As simulações computacionais para a análise do desempenho térmico do objeto de estudo foram realizadas no software EnergyPlus 8.9. As zonas térmicas foram modeladas no plugin Euclid para Sketchup e depois importadas no EnergyPlus. A residência foi dividida em 15 zonas térmicas, sendo que o ambiente estudado foi modelado como uma zona térmica única (Figura 7). Foram desenhados brises equivalentes e admitiu-se que as paredes externas e janelas estão desobstruídas do entorno.

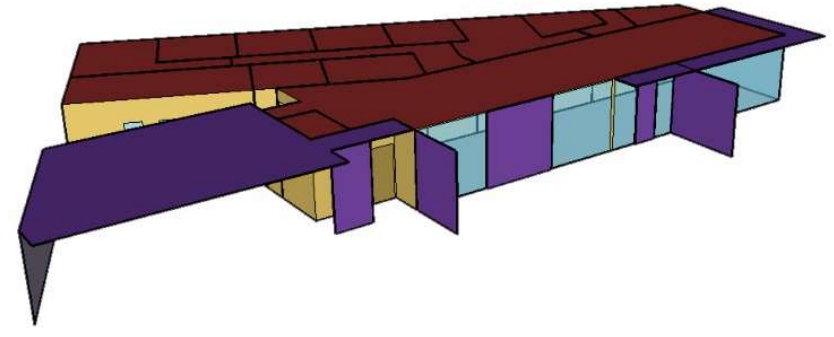

Figura 7- Zonas térmicas e brises modelados no Sketchup, através do plugin Euclid.

Fonte: produzido pelos autores.

Para a simulação da ventilação natural no EnergyPlus foi utilizado o grupo 'Airflow Network' e foi definida a área efetiva de abertura das janelas em $50 \%$ no período de ocupação do ambiente. As condições de utilização do ambiente e de ganhos térmicos foram configuradas segundo as indicações da Instrução Normativa Inmetro para a Classe de Eficiência Energética de Edificações Residenciais (CB3E, 2018). A ocupação foi configurada para duas 
pessoas das 14-18h e para quatro pessoas das 19-22h, com uma taxa metabólica definida em 108W. A iluminação artificial foi configurada para funcionar das $16-22 \mathrm{~h}$ com geração de $5 \mathrm{~W} / \mathrm{m}^{2}$ de calor e os equipamentos elétricos das 14-22h com potência total de 120W. Para o cálculo de troca de calor com o solo foi utilizado o processador Slab, no grupo 'Ground Heat Transfer'. Para os estudos realizados nesse trabalho foram pedidos como 'Outputs' no EnergyPlus apenas as variáveis 'Zone Operative Temperature' e 'Site Outdoor Air Drybulb Temperature'.

\subsection{Características dos materiais construtivos analisados}

As composições construtivas utilizadas nas simulações são descritas nos tópicos dos resultados, junto com sua transmitância térmica e capacidade térmica. As transmitâncias térmicas dos componentes construtivos foram retiradas do EnergyPlus, através do grupo 'Output: Table: Summary Reports' e a capacidade térmica foi calculada conforme as instruções presentes na NBR 15220-2/2005, cuja fórmula está representada abaixo. As propriedades térmicas dos materiais foram retiradas da mesma norma e estão representadas na Tabela 1.

Fórmula para cálculo da $\mathrm{Ct}=$ Capacidade térmica total capacidade térmica: $\mathrm{Ct}=$ e.c. $\rho$ e $=$ espessura da camada [m] $c=$ calor específico do material da camada $[\mathrm{J} / \mathrm{kg} . \mathrm{K}]$ $\rho=$ densidade de massa aparente do material da camada $\left[\mathrm{kg} / \mathrm{m}^{3}\right]$

Tabela 1- Propriedades Térmicas dos Materiais Utilizados nas Composições Construtivas.

\begin{tabular}{|c|c|c|c|c|c|}
\hline Material & $\begin{array}{c}\text { Condutividade } \\
[\mathbf{\Lambda}] \mathbf{( W / m} \cdot \mathbf{K})\end{array}$ & $\begin{array}{c}\text { Densidade } \\
{[\mathbf{[}] \mathbf{( k g / \mathbf { m } ^ { 3 } )}}\end{array}$ & $\begin{array}{c}\text { Calor } \\
\text { Específico } \\
{[\mathbf{c}] \mathbf{( J / k g . K )}}\end{array}$ & $\begin{array}{c}\text { Emissividade } \\
{[\mathbf{\varepsilon}]}\end{array}$ & $\begin{array}{c}\text { Absortividade } \\
{[\mathbf{\alpha}]}\end{array}$ \\
\hline $\begin{array}{c}\text { Argamassa Pintada de } \\
\text { Branco }\end{array}$ & 1,15 & 2000 & 1000 & 0,9 & 0,2 \\
\hline $\begin{array}{c}\text { Placa de Gesso } \\
\text { Acartonado }\end{array}$ & 0,35 & 875 & 840 & 0,9 & 0,2 \\
\hline Alumínio Telha & 201 & 2700 & 920 & 0,5 & 0,4 \\
\hline EPS & 0,04 & 25 & 1420 & 0,9 & 0,5 \\
\hline Lã de Rocha & 0,045 & 110 & 750 & 0,9 & 0,5 \\
\hline Concreto & 1,75 & 2200 & 1000 & 0,9 & 0,7 \\
\hline Piso de Madeira & 0,15 & 600 & 1340 & 0,9 & 0,7 \\
\hline Cerâmica & 0,9 & 1600 & 920 & 0,9 & 0,7 \\
\hline
\end{tabular}

Fonte: NBR 15220-2/2005. 


\subsection{Modelo Adaptativo para Ambientes Naturalmente Ventilados}

Foi utilizado o modelo adaptativo para ambientes naturalmente ventilados, proposto por DeDear e Brager (2002) e incorporado na ASHRAE55 de 2004. A equação para a determinação da temperatura de neutralidade proposta por esses autores está apresentada abaixo. Foi adotado o limite de conforto para $80 \%$ da população, somando e subtraindo $3,5^{\circ}$ na temperatura neutra para encontrar os limites máximos e mínimos de conforto, para cada mês do ano.

$$
\operatorname{Tn}=0,31 .(\text { Tmme })+17,8
$$

\section{RESULTADOS}

\subsection{Graus-hora de Resfriamento e Aquecimento do Projeto Original}

A simulação inicial do projeto teve como intuito encontrar a quantidade de graus-hora de resfriamento e aquecimento para a zona térmica da sala de estar, para o período de ocupação (14-22h). Os resultados das simulações demonstraram uma quantidade anual de 2.364,8 graus-hora de resfriamento e de 209,3 graus-hora de aquecimento, totalizando 2.574,1 graus-hora de desconforto. Esses dados apontam que a quantidade de graus-hora de resfriamento é mais de onze vezes superior à quantidade de graus-hora de aquecimento, evidenciando que o maior problema de desconforto é por calor. A Figura 8 representa a quantidade de graus-hora de resfriamento e aquecimento por mês, evidenciando que o mês de outubro é o que possui mais graus-horas de resfriamento, totalizando $27,4 \%$, e o mês de julho o que mais possui graushora de aquecimento, totalizando 49,2\%. A Figura 9 representa a distribuição anual dos graus-hora por hora do dia, com o período das 14-19h concentrando a maior carga de graus-hora de resfriamento, totalizando $97,6 \%$, e o período das 20-22h concentrando a maior carga de graus-hora de aquecimento, totalizando $83,3 \%$. 


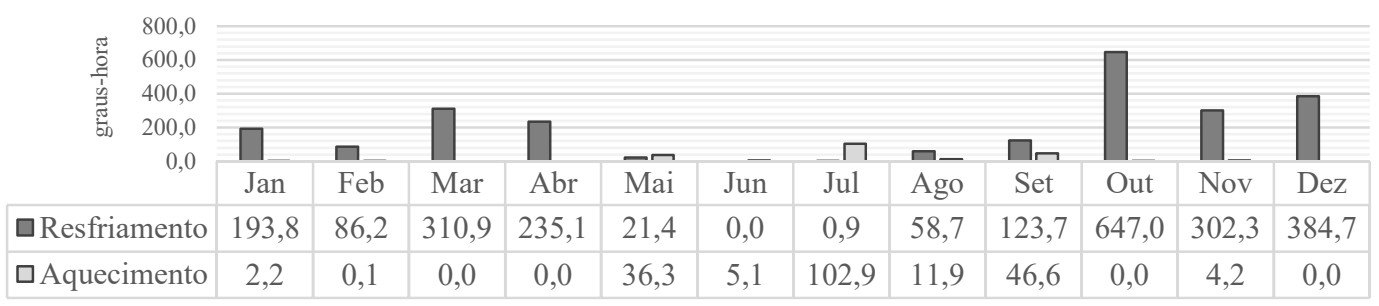

Figura 8- Distribuição dos graus-hora de desconforto por mês. Fonte: Simulações no EnergyPlus.

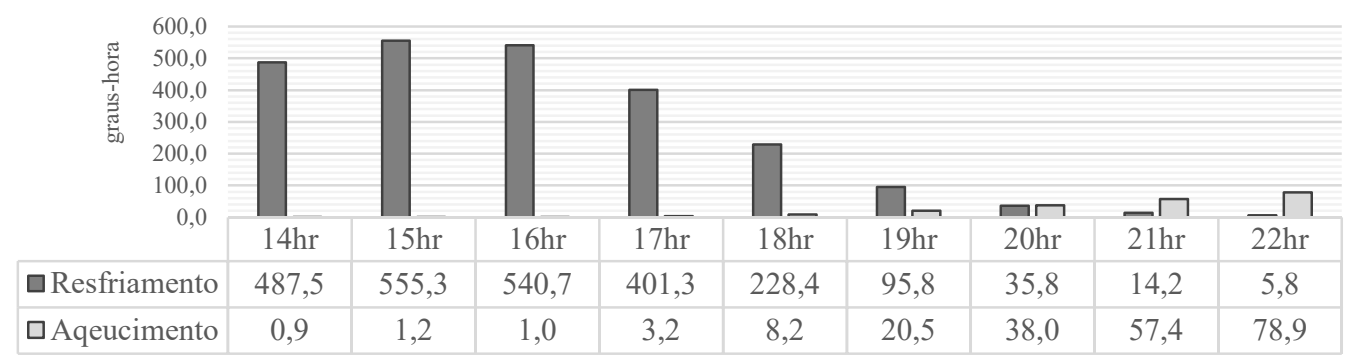

Figura 9- Distribuição dos graus-hora anuais de desconforto por hora do dia. Fonte:

Simulações no EnergyPlus.

\subsection{Cobertura}

Foram feitas oito simulações modificando os materiais e as camadas que compõem a cobertura (Tabela 2). Todas as simulações apresentaram redução do total de graus-hora de desconforto em comparação com o projeto original. A simulação 'Cob1' é a que demonstrou melhor eficiência térmica, com redução de $25,8 \%$ nos graus-hora de resfriamento e de $11,2 \%$ nos de aquecimento. Entretanto, observando os valores de resfriamento e aquecimento separadamente, a 'Cob3' é a solução que apresenta a maior redução dos graushora de resfriamento $(29,9 \%)$ e a 'Cob6' é a que apresenta a maior redução de graus-hora de aquecimento (19,3\%) (Figura 10). Nota-se que a 'Cob1' e 'Cob6' possuem o menor valor de transmitância térmica e o maior valor de capacidade térmica, já a 'Cob3' possui o maior valor de transmitância térmica e o menor de capacidade térmica, evidenciando que, ao analisar separadamente, a diminuição dos graus-hora de resfriamento do projeto está mais associada a sistemas de cobertura com transmitância térmica mais alta e capacidade térmica mais baixa, sendo o contrário para a diminuição dos graus-hora de aquecimento e dos graushora de resfriamento e aquecimento somados. 
Tabela 2- Descrição dos materiais utilizados em cada simulação de cobertura.

\begin{tabular}{|c|c|c|c|c|}
\hline Simulação & Composição & $\begin{array}{c}\text { Diferença do Projeto } \\
\text { Original }\end{array}$ & $\begin{array}{c}U \\
\left(W / m^{2} . K\right)\end{array}$ & $\begin{array}{c}C \\
\left(\mathrm{~kJ} / \mathrm{m}^{2} . K\right)\end{array}$ \\
\hline P. Original & $\begin{array}{l}\text { Telha Sanduíche }(\varepsilon=0,5 \alpha=0,4)+ \\
\text { Câmara } \operatorname{Ar}(\varepsilon>0,8)+\text { Lã de Rocha }(4 \mathrm{~cm}) \\
\text { + Forro de Gesso }(1,25 \mathrm{~cm})\end{array}$ & Original & 0,74 & 15,1 \\
\hline Cob1 & $\begin{array}{l}\text { Telha Sanduíche }(\varepsilon=0,9 \alpha=0,2)+ \\
\text { Câmara } \operatorname{Ar}(\varepsilon<0,2)+\text { Lã de Rocha } \\
(20 \mathrm{~cm})+\text { Forro de Gesso }(1,25 \mathrm{~cm})\end{array}$ & $\begin{array}{c}\text { Telha Pintada de Branca / } \\
\text { Lã de Rocha } 20 \mathrm{~cm} / \\
\text { Câmara } \operatorname{Ar} \varepsilon<0,2 \\
\end{array}$ & 0,19 & 28,3 \\
\hline Cob2 & $\begin{array}{l}\text { Telha Sanduíche }(\varepsilon=0,9 \alpha=0,2)+ \\
\text { Câmara } \operatorname{Ar}(\varepsilon>0,8)+\text { Lã de Rocha } \\
(20 \mathrm{~cm})+\text { Forro de Gesso }(1.25 \mathrm{~cm})\end{array}$ & $\begin{array}{l}\text { Telha Pintada de Branca / } \\
\text { Lã de Rocha } 20 \mathrm{~cm}\end{array}$ & 0,20 & 28,3 \\
\hline Cob3 & $\begin{array}{c}\text { Telha Sanduíche }(\varepsilon=0,9 \alpha=0,2)+ \\
\text { Câmara } \operatorname{Ar}(\varepsilon>0,8)+\text { Forro de Gesso } \\
(1,25 \mathrm{~cm})\end{array}$ & $\begin{array}{c}\text { Telha Pintada de Branca / } \\
\text { Sem Lã de Rocha }\end{array}$ & 2,18 & 11,8 \\
\hline Cob4 & $\begin{array}{c}\text { Telha Sanduíche }(\varepsilon=0,9 \alpha=0,2)+ \\
\text { Câmara } \operatorname{Ar}(\varepsilon<0,2)+\text { Forro de Gesso } \\
(1.25 \mathrm{~cm})\end{array}$ & $\begin{array}{c}\text { Telha Pintada de Branca / } \\
\text { Sem Lã de Rocha / } \\
\text { Câmara Ar } \varepsilon<0,2 \\
\end{array}$ & 1,17 & 11,8 \\
\hline Cob5 & $\begin{array}{c}\text { Telha Sanduíche }(\varepsilon=0,9 \alpha=0,2)+ \\
\text { Câmara } \operatorname{Ar}(\varepsilon>0,8)+\text { Lã de Rocha }(4 \mathrm{~cm}) \\
+ \text { Forro de Gesso }(1,25 \mathrm{~cm})\end{array}$ & Telha Pintada de Branca & 0,74 & 15,1 \\
\hline Cob6 & $\begin{array}{l}\text { Telha Sanduíche }(\varepsilon=0,5 \alpha=0,4)+ \\
\text { Câmara } \operatorname{Ar}(\varepsilon>0,8)+\text { Lã de Rocha } \\
(20 \mathrm{~cm})+\text { Forro de Gesso }(1,25 \mathrm{~cm})\end{array}$ & Lã de Rocha $20 \mathrm{~cm}$ & 0,20 & 28,3 \\
\hline Cob7 & $\begin{array}{l}\text { Telha Sanduíche }(\varepsilon=0,5 \alpha=0,4)+ \\
\text { Câmara } \operatorname{Ar}(\varepsilon>0,8)+\text { Lã de Rocha } \\
(10 \mathrm{~cm})+\text { Forro de Gesso }(1,25 \mathrm{~cm})\end{array}$ & Lã de Rocha $10 \mathrm{~cm}$ & 0,37 & 20,0 \\
\hline Cob8 & $\begin{array}{l}\text { Telha Sanduíche }(\varepsilon=0,5 \alpha=0,4)+ \\
\text { Câmara } \operatorname{Ar}(\varepsilon<0,2)+\text { Lã de Rocha }(4 \mathrm{~cm}) \\
+ \text { Forro de Gesso }(1,25 \mathrm{~cm})\end{array}$ & Câmara $\operatorname{Ar} \varepsilon<0,2$ & 0,57 & 15,1 \\
\hline
\end{tabular}

Fonte: Simulações no EnergyPlus e NBR 15220-2/2005.

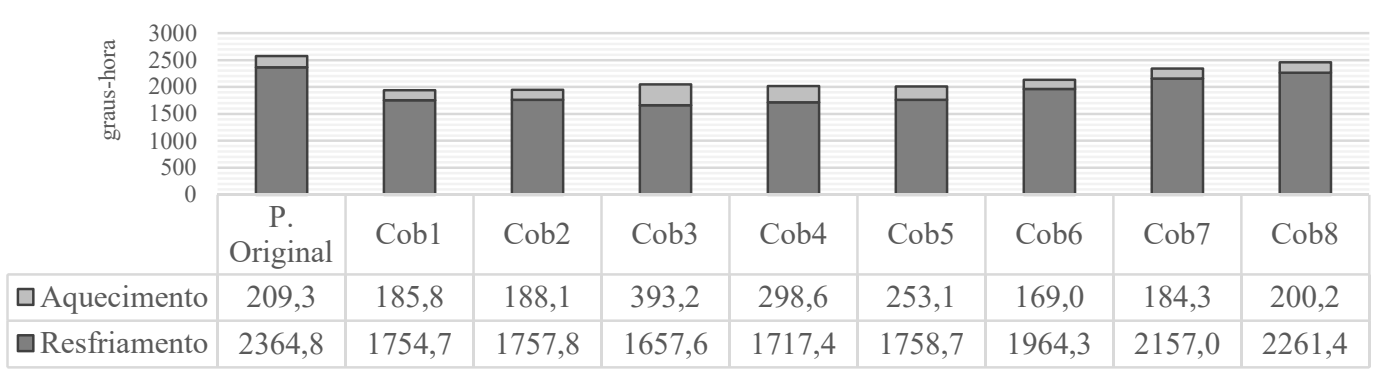

Figura 10- Quantificação dos graus-hora de resfriamento e aquecimento por simulação de cobertura. Fonte: Simulações no EnergyPlus.

\subsection{Piso}

Foram realizadas três simulações modificando os materiais e as camadas que compõem o piso (Tabela 3). Todas as simulações apresentaram redução do total de graus-hora de desconforto em comparação com o projeto 
original, sendo a simulação 'Piso1' a que teve a maior redução (Figura 11). As simulações 'Piso1' e 'Piso2' demonstraram uma redução nos graus-hora de resfriamento, $39,3 \%$ e $20,5 \%$ respectivamente, entretanto apresentaram um aumento nos graus-hora de aquecimento, $17,5 \%$ e $6,2 \%$, o que pode ser explicado pela ausência do piso de madeira e o consequente aumento da transmitância térmica do piso, favorecendo a troca de calor com o solo, que é mais frio.

Tabela 3- Descrição das composições utilizados em cada simulação de piso.

\begin{tabular}{|c|c|c|c|c|}
\hline Simulação & Composição & $\begin{array}{c}\text { Diferença do Projeto } \\
\text { Original }\end{array}$ & $\underset{\left(W / m^{2} . K\right)}{U}$ & $\begin{array}{c}C \\
\left(\mathrm{~kJ} / \mathrm{m}^{2} . K\right)\end{array}$ \\
\hline P. Original & $\begin{array}{c}\text { Laje de concreto }(20,0 \mathrm{~cm})+\text { Piso de } \\
\text { madeira }(2,5 \mathrm{~cm})\end{array}$ & Original & 2,22 & 460,1 \\
\hline Piso1 & Laje de concreto $(10,0 \mathrm{~cm})$ & $\begin{array}{l}\text { Redução de } 10,0 \mathrm{~cm} \text { de } \\
\text { laje de concreto e sem } \\
\text { piso de madeira }\end{array}$ & 3,52 & 220,0 \\
\hline Piso2 & Laje de concreto $(20,0 \mathrm{~cm})$ & Sem piso de madeira & 3,52 & 440,0 \\
\hline Piso3 & $\begin{array}{l}\text { Laje de concreto }(10,0 \mathrm{~cm})+\text { Piso de } \\
\text { madeira }(2,5 \mathrm{~cm})\end{array}$ & $\begin{array}{l}\text { Redução de } 10,0 \mathrm{~cm} \text { de } \\
\text { laje de concreto }\end{array}$ & 2,54 & 240,1 \\
\hline
\end{tabular}

Fonte: Simulações no EnergyPlus e NBR 15220-2/2005.

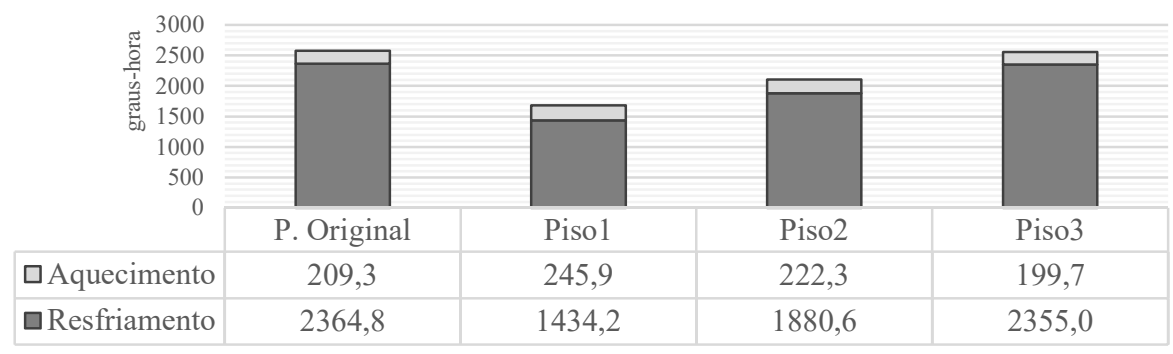

Figura 11- Quantificação dos graus-hora de resfriamento e aquecimento por simulação de piso. Fonte: Simulações no EnergyPlus.

\subsection{Vidros}

Foram realizadas três simulações modificando os tipos dos vidros utilizados (Tabela 4). Todas as simulações apresentaram redução do total de graus-hora de desconforto em comparação com o projeto original. A simulação 'Vidro1' apresentou a maior redução, tanto dos graus-hora de resfriamento $(42,0 \%)$ como dos graus-hora de aquecimento (15,4\%) (Figura 12). As simulações 'Vidro2' e 'Vidro3' demonstraram uma redução nos graus-hora de 
resfriamento, $15,8 \%$ e $11,3 \%$ respectivamente, mas apresentaram um aumento nos graus-hora de aquecimento, 22,1\% e 18,6\%. Nota-se que a diminuição do fator solar e da transmissão da luz visível favorecem a diminuição dos graushora de resfriamento, mas quando essa redução não é acompanhada pela redução da transmitância térmica os graus-hora de aquecimento aumentaram, pois diminui-se o ganho de calor por radiação solar, mas mantem-se a mesma transmissão de calor por condução e convecção.

Tabela 4- Descrição das composições utilizadas em cada simulação de vidro.

\begin{tabular}{|c|c|c|c|c|}
\hline Simulação & Composição & $\begin{array}{c}\mathbf{U} \\
\left(\mathbf{W} / \mathbf{m}^{\mathbf{2}} \cdot \mathbf{K}\right)\end{array}$ & $\begin{array}{c}\text { Fator } \\
\text { Solar }\end{array}$ & $\begin{array}{c}\mathbf{T} \text {. Luz } \\
\text { Visível }\end{array}$ \\
\hline P. Original & Temperado incolor 8mm & 5,60 & 0,87 & 0,83 \\
\hline Vidro1 & $\begin{array}{c}\text { Insulado (Incolor 6 mm com controle solar face 2 + ar 12 } \\
\mathrm{mm}+\text { incolor 6 mm) }\end{array}$ & 1,60 & 0,50 & 0,28 \\
\hline Vidro2 & Laminado (Incolor 4 mm com controle solar na face 2 + \\
incolor 4 mm) & 5,60 & 0,51 & 0,34 \\
\hline Vidro3 & Verde 8mm & 5,60 & 0,60 & 0,73 \\
\hline
\end{tabular}

Fonte: Simulações no EnergyPlus e NBR 15220-2/2005.

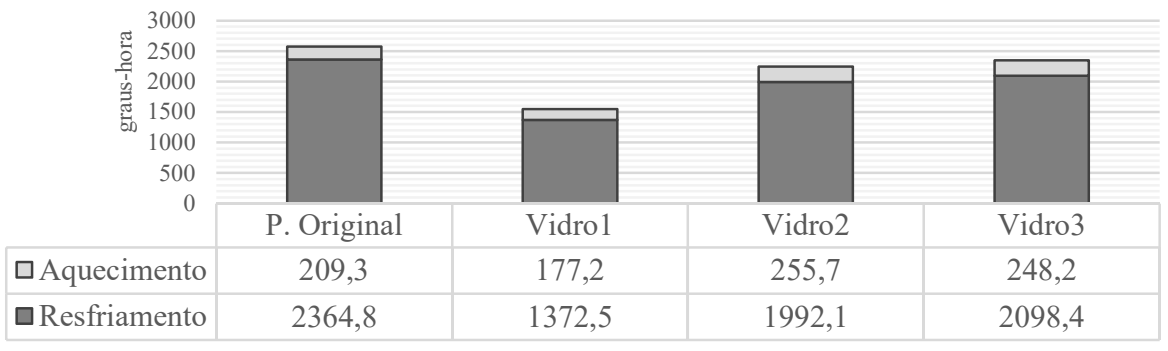

Figura 12- Quantificação dos graus-hora de resfriamento e aquecimento por simulação de vidro. Fonte: Simulações no EnergyPlus.

\subsection{Paredes Internas}

Foi realizada uma simulação para as paredes internas do projeto, utilizando paredes duplas (Tabela 5). A simulação demonstrou que houve um pequeno aumento do total de graus-hora de desconforto em comparação ao projeto original, aproximadamente $2 \%$ (Figura 13). Analisando os valores de resfriamento e aquecimento separadamente, observa-se que houve um aumento de $2,6 \%$ da quantidade de graus-hora de resfriamento e uma redução de 5,5\% de graus-hora de aquecimento, já que a diminuição da transmitância térmica e o 
aumento da capacidade térmica das paredes internas resultaram em uma menor e mais lenta troca de calor entre os ambientes, retendo mais calor dentro da sala.

Tabela 5- Descrição das composições utilizadas na simulação de paredes internas.

\begin{tabular}{|c|c|c|c|c|}
\hline Simulação & Composição & $\begin{array}{c}\text { Diferença do } \\
\text { Projeto } \\
\text { Original }\end{array}$ & $\begin{array}{c}\mathbf{U} \\
\left(\mathbf{W} / \mathbf{m}^{\mathbf{2}} . \mathbf{K}\right)\end{array}$ & $\begin{array}{c}\mathbf{C} \\
\left.\mathbf{( k J} / \mathbf{m}^{\mathbf{2}} . \mathbf{K}\right)\end{array}$ \\
\hline P. Original & $\begin{array}{c}\text { Bloco de Cerâmica }(14 \times 19 \times 29 \mathrm{~cm})+\text { Argamassa } \\
(2,5+2,5 \mathrm{~cm} \varepsilon=009 \alpha=0,2)\end{array}$ & Original & 2,01 & 148,8 \\
\hline $\begin{array}{c}\text { Parede } \\
\text { Dupla }\end{array}$ & $\begin{array}{c}2 \times \text { Bloco de Cerâmica }(14 \times 19 \times 29 \mathrm{~cm})+ \\
\text { Argamassa }(2,5+2,5 \mathrm{~cm} \varepsilon=0,9 \alpha=0,2)\end{array}$ & Parede Dupla & 1,25 & 197,6 \\
\hline
\end{tabular}

Fonte: Simulações no EnergyPlus e NBR 15220-2/2005.

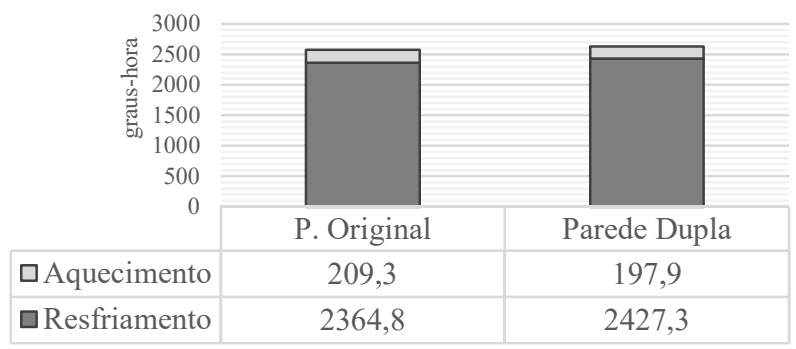

Figura 13- Quantificação dos graus-hora de resfriamento e aquecimento para a simulação de paredes internas. Fonte: Simulações no EnergyPlus.

\subsection{Proteções Solares}

Foram realizadas nove simulações com as proteções solares do projeto, variando o ângulo ${ }^{3}$ alfa dos beirais, o ângulo beta dos brises verticais, a quantidade de área sombreada por elemento frontal à janela e inserindo um brise vertical do lado direito da janela da face sul (Tabela 6). A simulação 'PS9' foi feita sem nenhuma proteção solar, apenas como base de comparação, evidenciando que sem os sombreamentos haveria um acréscimo de $23,1 \%$ de graus-hora de desconforto. As demais simulações demonstram que quanto mais sombreadas as janelas, ou seja, quanto melhor dimensionados os sistemas de proteção, menor a quantidade de graus-hora de resfriamento e maior a de aquecimento, devido à menor quantidade de radiação transmitida para dentro do ambiente. Exemplificando, temos a simulação 'PS1', com o maior sombreamento, que

\footnotetext{
${ }^{3}$ Foram utilizados os ângulos internos.
} 
reduziu em $32,9 \%$ a quantidade de graus-hora de resfriamento e aumentou em $13,8 \%$ a de aquecimento, e a simulação 'PS8', que reduziu apenas 1,6\% a quantidade de graus-hora de resfriamento e aumentou apenas $0,5 \%$ a de aquecimento (Figura 14). A divisão das faces está exemplificada na Figura 6.

Tabela 6- Descrição das composições utilizadas nas simulações de proteção solar.

\begin{tabular}{|c|c|c|c|c|}
\hline Simulação & $\begin{array}{c}\text { Face Oeste } 1 \\
(48 \% \text { da abertura) }\end{array}$ & $\begin{array}{c}\text { Face Oeste } 2 \\
(17 \% \text { da abertura) }\end{array}$ & $\begin{array}{c}\text { Face Oeste } 3 \\
\text { (35\% da abertura) }\end{array}$ & $\begin{array}{l}\text { Face Sul } \\
(100 \% \text { da } \\
\text { abertura) }\end{array}$ \\
\hline P. Original & $\begin{array}{c}\text { Somb. Frontal }(33 \%) \\
\text { Brise Vertical } \\
\left(\beta=11,3^{\circ}\right)\end{array}$ & $\begin{array}{c}\text { Beiral } 120 \mathrm{~cm} \\
\left(\alpha=21.8^{\circ}\right) \text { Somb. } \\
\text { Frontal }(33 \%) \text { Brise } \\
\text { Vertical }\left(\beta=11,3^{\circ}\right)\end{array}$ & $\begin{array}{l}\text { Beiral } 120 \mathrm{~cm} \\
\qquad\left(\alpha=21,8^{\circ}\right)\end{array}$ & $\begin{array}{l}\text { Beiral } 120 \mathrm{~cm} \\
\qquad\left(\alpha=21,8^{\circ}\right)\end{array}$ \\
\hline PS1 & $\begin{array}{c}\text { Beiral } 180 \mathrm{~cm} \\
\left(\alpha=31,1^{\circ}\right) \text { Somb. } \\
\text { Frontal }(33 \%) \text { Brise } \\
\text { Vertical }\left(\beta=11,3^{\circ}\right)\end{array}$ & $\begin{array}{c}\text { Beiral } 180 \mathrm{~cm} \\
\left(\alpha=31,1^{\circ}\right) \text { Somb. } \\
\text { Frontal }(33 \%) \text { Brise } \\
\text { Vertical }\left(\beta=11,3^{\circ}\right)\end{array}$ & $\begin{array}{c}\text { Beiral } 180 \mathrm{~cm} \\
\left(\alpha=31,1^{\circ}\right) \text { Somb. } \\
\text { Frontal }(33 \%) \text { Brise } \\
\text { Vertical }\left(\beta=11,3^{\circ}\right)\end{array}$ & $\begin{array}{l}\text { Beiral } 120 \mathrm{~cm} \\
\left(\alpha=21,8^{\circ}\right) \\
\text { Brise Vertical } \\
\left(\beta d=28,8^{\circ}\right)\end{array}$ \\
\hline PS2 & $\begin{array}{c}\text { Beiral } 120 \mathrm{~cm} \\
\left(\alpha=21,8^{\circ}\right) \text { Somb. } \\
\text { Frontal }(33 \%) \text { Brise } \\
\text { Vertical }\left(\beta=11,3^{\circ}\right)\end{array}$ & $\begin{array}{c}\text { Beiral } 120 \mathrm{~cm} \\
\left(\alpha=21,8^{\circ}\right) \text { Somb. } \\
\text { Frontal }(33 \%) \text { Brise } \\
\text { Vertical }\left(\beta=11,3^{\circ}\right)\end{array}$ & $\begin{array}{c}\text { Beiral } 120 \mathrm{~cm} \\
\left(\alpha=21,8^{\circ}\right) \text { Somb. } \\
\text { Frontal }(33 \%) \text { Brise } \\
\text { Vertical }\left(\beta=11,3^{\circ}\right)\end{array}$ & $\begin{array}{l}\text { Beiral } 120 \mathrm{~cm} \\
\left(\alpha=21,8^{\circ}\right) \\
\text { Brise Vertical } \\
\left(\beta d=28.8^{\circ}\right)\end{array}$ \\
\hline PS3 & $\begin{array}{c}\text { Beiral } 240 \mathrm{~cm} \\
\left(\alpha=38.8^{\circ}\right) \text { Somb. } \\
\text { Frontal }(33 \%) \text { Brise } \\
\text { Vertical }\left(\beta=11.3^{\circ}\right)\end{array}$ & $\begin{array}{c}\text { Beiral } 240 \mathrm{~cm} \\
\left(\alpha=38.8^{\circ}\right) \text { Somb. } \\
\text { Frontal }(33 \%) \text { Brise } \\
\text { Vertical }\left(\beta=11.3^{\circ}\right)\end{array}$ & $\begin{array}{l}\text { Beiral } 240 \mathrm{~cm} \\
\qquad\left(\alpha=38.8^{\circ}\right)\end{array}$ & $\begin{array}{l}\text { Beiral } 120 \mathrm{~cm} \\
\qquad\left(\alpha=21.8^{\circ}\right)\end{array}$ \\
\hline PS4 & $\begin{array}{c}\text { Beiral } 180 \mathrm{~cm} \\
\left(\alpha=31.1^{\circ}\right) \text { Somb. } \\
\text { Frontal }(33 \%) \text { Brise } \\
\text { Vertical }\left(\beta=11.3^{\circ}\right)\end{array}$ & $\begin{array}{c}\text { Beiral } 180 \mathrm{~cm} \\
\left(\alpha=31.1^{\circ}\right) \text { Somb. } \\
\text { Frontal }(33 \%) \text { Brise } \\
\text { Vertical }\left(\beta=11.3^{\circ}\right) \\
\end{array}$ & $\begin{array}{l}\text { Beiral } 180 \mathrm{~cm} \\
\qquad\left(\alpha=31.1^{\circ}\right)\end{array}$ & $\begin{array}{l}\text { Beiral } 120 \mathrm{~cm} \\
\qquad\left(\alpha=21.8^{\circ}\right)\end{array}$ \\
\hline PS5 & $\begin{array}{c}\text { Somb. Frontal }(33 \%) \\
\text { Brise Vertical } \\
\left(\beta=11.3^{\circ}\right)\end{array}$ & $\begin{array}{c}\text { Beiral } 120 \mathrm{~cm} \\
\left(\alpha=21.8^{\circ}\right) \text { Somb. } \\
\text { Frontal }(33 \%) \text { Brise } \\
\text { Vertical }\left(\beta=11.3^{\circ}\right) \\
\end{array}$ & $\begin{array}{c}\text { Beiral } 120 \mathrm{~cm} \\
\left(\alpha=21.8^{\circ}\right) \text { Somb. } \\
\text { Frontal }(33 \%) \text { Brise } \\
\text { Vertical }\left(\beta=11.3^{\circ}\right) \\
\end{array}$ & $\begin{array}{l}\text { Beiral } 120 \mathrm{~cm} \\
\qquad\left(\alpha=21.8^{\circ}\right)\end{array}$ \\
\hline PS6 & $\begin{array}{c}\text { Beiral } 120 \mathrm{~cm} \\
\left(\alpha=21.8^{\circ}\right) \text { Somb. } \\
\text { Frontal }(33 \%) \text { Brise } \\
\text { Vertical }\left(\beta=11.3^{\circ}\right)\end{array}$ & $\begin{array}{c}\text { Beiral } 120 \mathrm{~cm} \\
\left(\alpha=21.8^{\circ}\right) \text { Somb. } \\
\text { Frontal }(33 \%) \text { Brise } \\
\text { Vertical }\left(\beta=11.3^{\circ}\right)\end{array}$ & $\begin{array}{l}\text { Beiral } 120 \mathrm{~cm} \\
\qquad\left(\alpha=21.8^{\circ}\right)\end{array}$ & $\begin{array}{l}\text { Beiral } 120 \mathrm{~cm} \\
\qquad\left(\alpha=21.8^{\circ}\right)\end{array}$ \\
\hline PS7 & $\begin{array}{l}\text { Somb. Frontal }(33 \%) \\
\text { Brise Vertical }\left(\beta=24^{\circ}\right)\end{array}$ & $\begin{array}{c}\text { Beiral } 120 \mathrm{~cm} \\
\left(\alpha=21.8^{\circ}\right) \text { Somb. } \\
\text { Frontal }(33 \%) \text { Brise } \\
\text { Vertical }\left(\beta=24^{\circ}\right)\end{array}$ & $\begin{array}{l}\text { Beiral } 120 \mathrm{~cm} \\
\quad\left(\alpha=21.8^{\circ}\right)\end{array}$ & $\begin{array}{l}\text { Beiral } 120 \mathrm{~cm} \\
\qquad\left(\alpha=21.8^{\circ}\right)\end{array}$ \\
\hline PS8 & $\begin{array}{c}\text { Somb. Frontal }(33 \%) \\
\text { Brise Vertical } \\
\left(\beta=11.3^{\circ}\right)\end{array}$ & $\begin{array}{c}\text { Beiral } 120 \mathrm{~cm} \\
\left(\alpha=21.8^{\circ}\right) \text { Somb. } \\
\text { Frontal }(33 \%) \text { Brise } \\
\text { Vertical }\left(\beta=11.3^{\circ}\right)\end{array}$ & $\begin{array}{l}\text { Beiral } 120 \mathrm{~cm} \\
\qquad\left(\alpha=21.8^{\circ}\right)\end{array}$ & $\begin{array}{l}\text { Beiral } 120 \mathrm{~cm} \\
\left(\alpha=21.8^{\circ}\right) \\
\text { Brise Vertical } \\
\left(\beta d=28.8^{\circ}\right)\end{array}$ \\
\hline PS9 & Sem sombreamento & Sem sombreamento & Sem sombreamento & $\begin{array}{c}\text { Sem } \\
\text { sombreamento }\end{array}$ \\
\hline
\end{tabular}

Fonte: Simulações no EnergyPlus e NBR 15220-2/2005. 


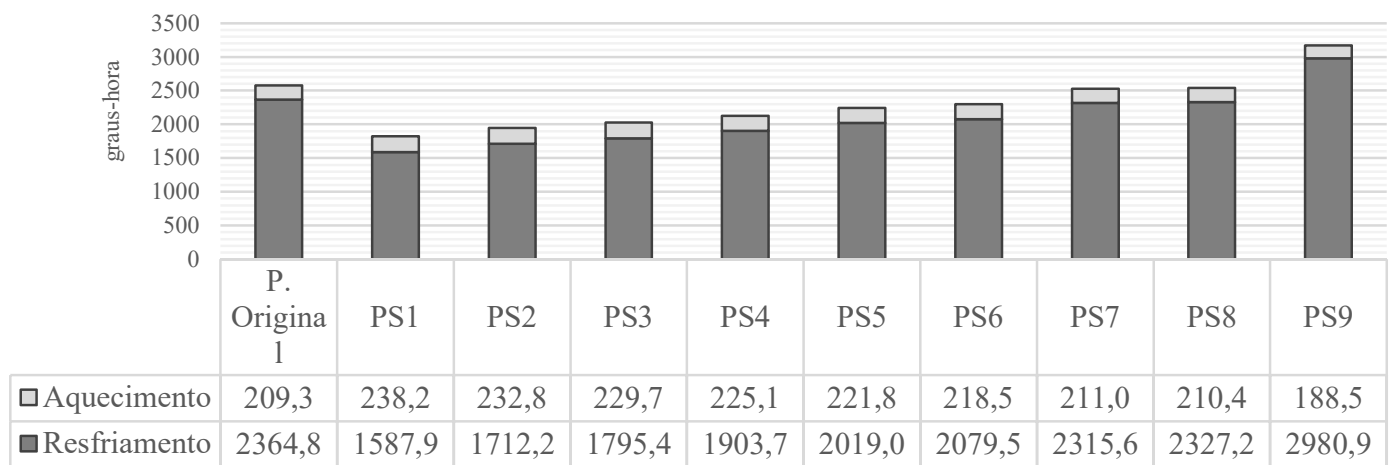

Figura 14- Quantificação dos graus-hora de resfriamento e aquecimento por simulação de proteção solar. Fonte: Simulações no EnergyPlus.

\subsection{Composições}

Esse tópico busca relacionar algumas das simulações feitas nos tópicos anteriores de forma conjunta. Foram realizadas três simulações, a ' $C 1$ ' reuniu as alterações que demonstraram mais redução de graus-hora de resfriamento, a 'C3' buscou modificar o mínimo possível o projeto original e a 'C2' é uma situação intermediária (Tabela 7). A primeira simulação foi a única que diminuiu os graushora de resfriamento e aquecimento conjuntamente, $89,2 \%$ e 18,3\% respectivamente. As outras duas simulações tiveram a redução apenas dos graus-hora de resfriamento, 'C2' com 74,2\% e 'C3' com 48,4\%, mas o aumento dos graus-hora de aquecimento, 'C2' com 10,3\% e 'C3' com 26,9\% (Figura 15). A diminuição dos graus-hora de aquecimento na primeira simulação ocorre principalmente por conta do uso do vidro insulado.

Tabela 7- Descrição dos elementos utilizados nas simulações de composições.

\begin{tabular}{|c|c|}
\hline Simulação & Composição \\
\hline P. Original & $\begin{array}{c}\text { [Telha Termoacústica Alumínio + Câmara de Ar } \varepsilon>0,8+\text { Lã de Rocha } 4 \mathrm{~cm}]+[\text { Piso } \\
\text { Concreto } 20 \mathrm{~cm}+\text { Piso de Madeira de } 2,5 \mathrm{~cm}]+[\text { Beiral de } 120 \mathrm{~cm} \text { nas Faces Oeste } 2 \text { e } 3 \\
\left.\text { com } \alpha=31,1^{\circ}\right]+\left[\text { Sombreamento Frontal nas Faces Oeste } 1 \text { e } 2 \text { com } \beta=11.3^{\circ}\right]+[\text { Vidro } \\
\text { Temperado] }\end{array}$ \\
\hline C1 & $\begin{array}{c}\text { [Telha Termoacústica Pintada de Branco + Câmara de Ar } \varepsilon<0,2+\text { Lã de Rocha } 20 \mathrm{~cm}]+ \\
\text { [Piso Concreto } 10 \mathrm{~cm}]+\left[\text { Beiral de } 180 \mathrm{~cm} \text { nas Faces Oeste } 1,2 \text { e } 3 \text { com } \alpha=31,1^{\circ}\right]+ \\
\left.\text { [Sombreamento Frontal nas Faces Oeste } 1,2 \text { e } 3 \text { com } \beta=11,3^{\circ}\right]+[\text { Brise Vertical Sul com } \\
\left.\beta d=28,8^{\circ}\right]+[\text { Vidro Insulado }]\end{array}$ \\
\hline $\mathrm{C} 2$ & $\begin{array}{c}\text { [Telha Termoacústica Pintada de Branco + Câmara de Ar } \varepsilon<0,2+\text { Lã de Rocha } 20 \mathrm{~cm}]+ \\
\text { [Piso Concreto } 10 \mathrm{~cm}]+\left[\text { Beiral de } 120 \mathrm{~cm} \text { nas Faces Oeste } 1,2 \text { e } 3 \text { com } \alpha=31,1^{\circ}\right]+ \\
\left.\text { [Sombreamento Frontal nas Faces Oeste } 1,2 \text { e } 3 \text { com } \beta=11,3^{\circ}\right]+[\text { Brise Vertical Sul com } \\
\left.\beta d=28,8^{\circ}\right]+[\text { Vidro Laminado] }\end{array}$ \\
\hline C3 & $\begin{array}{c}\text { [Telha Termoacústica Pintada de Branco + Câmara de Ar } \varepsilon>0,8+\text { Lã de Rocha } 4 \mathrm{~cm}]+ \\
\text { [Piso Concreto } 10 \mathrm{~cm}+\text { Piso de Madeira de } 2,5 \mathrm{~cm}]+[\text { Beiral de } 120 \mathrm{~cm} \text { nas Faces Oeste } \\
\left.1,2 \text { e } 3 \text { com } \alpha=31,1^{\circ}\right]+\left[\text { Sombreamento Frontal nas Faces Oeste } 1,2 \text { e com } \beta=11,3^{\circ} \text { ] }\right. \\
\text { [Vidro Temperado] }\end{array}$ \\
\hline
\end{tabular}


Fonte: Simulações no EnergyPlus e NBR 15220-2/2005.

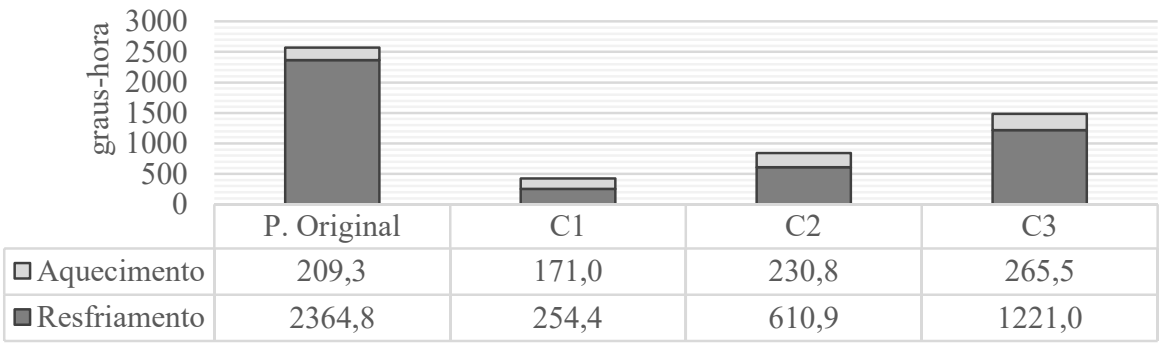

Figura 15- Quantificação dos graus-hora de resfriamento e aquecimento por simulação de composição. Fonte: Simulações no EnergyPlus.

\section{CONCLUSÕES}

A utilização do modelo adaptativo para ambientes naturalmente ventilados da ASHRAE55 em paralelo com as simulações desenvolvidas no Energy Plus demonstrou ser um método útil e de fácil aplicação tanto para a análise da eficiência térmica do projeto, como para balizar o estudo e a escolha de materiais de construção e de estratégias passivas de conforto. A aplicação desse método no projeto estudado apontou a quantidade de graus-hora de desconforto na situação original do projeto e permitiu que fossem feitas comparações com as simulações alternativas, demonstrando quantitativamente o aumento ou a redução de graus-hora de resfriamento e aquecimento.

As simulações realizadas nesse trabalho foram tematizadas com 0 intuito de entender como cada alteração modificaria a quantidade de graus-hora de desconforto do projeto original. O primeiro tema foi cobertura e as simulações demonstraram que pequenas alterações nos materiais já trariam mais conforto ao projeto, por exemplo, ao pintar as telhas de branco (baixar a absortância) houve uma redução de $21,8 \%$ dos graus-hora de desconforto e, se somado com a colocação de um material com alta emissividade na câmara de ar e com o aumento de $4 \mathrm{~cm}$ para $20 \mathrm{~cm}$ na espessura da lã de rocha, atingiria $24,6 \%$ de redução na carga de desconforto. Outro dado interessante dessa temática foi que ao olhar apenas para a redução dos graus-hora de resfriamento observa-se que sistemas de cobertura com maior transmitância térmica e menor capacidade térmica tiveram maior redução na quantidade de graus-hora, como por exemplo, a cobertura pintada de branco, sem lã de rocha e sem material com alta 
emissividade na câmara de ar reduziu $29,9 \%$ da carga de resfriamento, entretanto não demonstrou ser eficaz para a carga de aquecimento, aumentando em $87,9 \%$.

No estudo dos pisos as simulações demonstraram que o aumento da transmitância térmica favorece consideravelmente a diminuição dos graus-hora de resfriamento, pois facilita a troca de calor do ambiente com o solo. A retirada do piso de madeira, que tem uma resistência térmica alta, e a diminuição de $50 \%$ da espessura da laje de concreto trouxe uma redução de $34,7 \%$ na carga de resfriamento, uma redução expressiva com a utilização de menos material de construção. Entretanto, com o aumento da transmitância térmica do piso há também o aumento da carga de aquecimento do ambiente, sendo necessário uma ponderação no uso dessa estratégia. No tópico dos vidros as simulações apontaram que apenas com a substituição dos vidros originais para os insulados a carga de desconforto reduziria em $39,8 \%$, uma redução expressiva alterando apenas um componente construtivo. Vale ressaltar que a diminuição do fator solar e da transmissão da luz visível dos vidros quando não acompanhada pela redução da transmitância térmica, como o caso dos vidros verdes e laminados, a carga de aquecimento tende a aumentar.

No estudo das paredes internas a simulação demonstrou que quando utilizadas paredes duplas a carga de desconforto aumentou em $2 \%$, ou seja, não foi uma estratégia eficaz para o aumento do período de conforto do ambiente. No tópico de proteções solares as simulações apontaram uma relação inversa entre o graus-hora de resfriamento e de aquecimento, ou seja, quanto melhor dimensionados os brises e beirais, menor a carga de resfriamento e maior a carga de aquecimento do ambiente, sendo necessário fazer uma ponderação conforme necessidades do projeto.

No tópico de composições as simulações demonstraram que podemos atingir bons resultados na diminuição dos graus-hora de desconforto com poucas modificações nos materiais e nas composições construtivas do projeto original. A simulação que menos alterou o projeto conseguiu reduzir em $42,2 \%$ a carga de desconforto, apenas pintando a telha de branco, diminuindo a laje de concreto do piso para $10 \mathrm{~cm}$ e estendendo o beiral e o brise original para toda a face oeste, ou seja, soluções com baixo custo que aumentariam consideravelmente 0 período de conforto do ambiente. A solução que mais alterou o projeto e contou 
com materiais mais custosos, como o vidro insulado, conseguiu reduzir expressivos $83,5 \%$ da carga de desconforto, mostrando que é possível reduzir drasticamente o período de desconforto do ambiente sem a utilização de sistemas ativos, como o ar condicionado por exemplo, mesmo em uma situação com orientação desfavorável, como o ambiente aqui estudado.

\section{REFERÊNCIAS BIBLIOGRÁFICAS}

ASHARE. Standard 55: Thermal Environment Conditions for Human Occupancy. Atlanta: American Society of Heating, Refrigerating and Air-Conditioning Engineers, 2004.

Associação Brasileira de Normas Técnicas. NBR 15220-2: Desempenho térmico de edificações - Parte 2: Métodos de cálculo da transmitância térmica, da capacidade térmica, do atraso térmico e do fator solar de elementos e componentes de edificações. Rio de Janeiro, 2005.

Centro Brasileiro de Eficiência Energética em Edificações (CB3E). Instrução normativa INMETRO para a Classe de Eficiência Energética de Edificações Residenciais. Florianopolis, 2018.

DEDEAR, R. J.; BRAGER, G. S. Thermal Comfort in Naturally Ventilated Buildings: revisions to ASHAE Standard 55. Energy and Buildings, v.34, n.6, p.549-561, jul. 2002.

PEREIRA, I. M.; ASSIS, E. S. Avaliação de modelos de índices adaptativos para uso no projeto arquitetônico bioclimático. Ambiente Construído, Porto Alegre, v. 10, n.1, p.31-51, jan./mar. 2010.

\section{SOBRE OS AUTORES}

Daniel Teixeira Turczyn - danieltturczyn@gmail.com

Arquiteto urbanista, doutor em Arquitetura, Tecnologia e Cidade pela Universidade Estadual de Campinas (UNICAMP). Atua como projetista, professor e pesquisador, com enfoque nos temas: projetos de arquitetura e urbanismo, morfologia e paisagem urbana, desenho e planejamento urbano, tecnologias e projetos sustentáveis e práticas de ensinagem em arquitetura e urbanismo. Integrante do grupo de pesquisa FLUXUS (Laboratório de Redes Técnicas e Sustentabilidade Socioambiental) da UNICAMP.

Letícia de Oliveira Neves - leticia@fec.unicamp.br

Arquiteta urbanista e professora doutora do Departamento de Arquitetura e Construção da Faculdade de Engenharia Civil, Arquitetura e Urbanismo (FEC), Universidade Estadual de Campinas (UNICAMP). Atua principalmente nos seguintes temas: arquitetura bioclimática, eficiência energética, conforto térmico e ventilação natural. Membro da diretoria do Chapter Brasil da ASHRAE e do IBPSA Brasil. Integrante do grupo de pesquisa do LaCAF (Laboratório de Conforto Ambiental e Física Aplicada) da UNICAMP. 